\title{
A Empatia Como um dos Pilares da Humanização da Relação Médico-Paciente. Evolução de Três Anos do Projeto "Calouro Humano"
}

\section{Empathy as one of the pillars of the humanization of the doctor-patient relationship. Three-Year Evolution of the "Calouro Humano" Project}

Sara Cristine Marques dos Santos ${ }^{\dagger}$, Lívia Liberata Barbosa Bandeira Lemos de Souza Macedoll, Eucir Rabello ${ }^{\circ}$, Ivana Picone Borges de Aragão ${ }^{a^{*}}$

Como citar esse artigo. Dos Santos,

S.C.M.; Bandeira, L.L.B.; Dos Anjos, I.L.P.B.; Macedo, T.L.S.; Rabello, E.; De Aragão, I.P.B. A Empatia Como um dos Pilares da Humanização da Relação Médico-Paciente. Revista de Saúde. 2020 Jan./Jun.; 11 (1): 49-54.

\section{Resumo}

O médico, na sua atuação, precisa demonstrar compaixão e ser solidário, respeitando e se solidarizando com o que lhe é apresentado, culminando no estabelecimento de uma agradável relação médico paciente (RMP). Essa relação será fundamental para que haja o desenvolvimento de um perfil de paciente colaborativo, onde possui maior confiança no médico e que estará sujeito a uma maior adesão aos tratamentos, desta forma, evoluindo melhor e com melhor aproveitamento terapêutico. O objetivo do presente estudo foi avaliar a humanização na relação médico-paciente em alunos do primeiro período de curso de medicina (APPCM). Projeto de pesquisa e extensão da Universidade de Vassouras, sob parecer do Conselho de Ética em Pesquisa (CEP) n 1.963.944, com início em 2017. Período de avaliação foi entre 2017 e 2019. A aproximação do APPM ao paciente foi realizada pelo auxílio do aluno mentor matriculado a partir do segundo período de medicina. Um total de 2386 pacientes e 779 alunos foram entrevistados através de questionários. A aproximação proporcionada pelo APPM aos pacientes, foi interpretada como positiva tanto pelos alunos como pelos pacientes. Foi possível observar que os estudantes de medicina foram capazes de participar das necessidades dos pacientes, auxiliando, cuidando, fornecendo informações durante a internação, trazendo mais conforto e tranquilidade aos pacientes durante a internação, procurando suprir problemas relatados pelos pacientes em várias áreas relatadas, trabalhando no estímulo da habilidade de comunicação. A introdução dos alunos de medicina precocemente ao paciente incentivou o fortalecimento do processo de humanização na relação médico-paciente.

Palavras-chave: Humanização da Assistência. Educação Médica. Estudantes de Medicina.

\begin{abstract}
In medical practice is necessary to demonstrate compassion, support, solidarity, respect, with the situations that may arise, culminating in the establishment of a pleasant doctor-patient relationship (RMP). This relationship will be essential for the development of a collaborative patient profile, where you have greater confidence in the doctor and who will be subject to greater adherence to treatments, thus, evolving better and with better therapeutic use. The present study aimed to evaluate humanization in the doctor-patient relationship in students of the first period of medical school (SFPMS). Research and extension project at the University of Vassouras, under the opinion of the Research Ethics Council from Brazil No. 1,963,944, starting in 2017. The evaluation period was between 2017 and 2019. The SFPMS approach to the patient was carried out by aid from the mentor-student enrolled in the second period of medicine onwards. A total of 2386 patients and 779 students were interviewed using questionnaires. The approach provided by the SFPMS to patients was interpreted as positive by both students and patients. It was possible to observe that medical students were able to participate in the patients' needs, assisting, caring, providing information during hospitalization, bringing more comfort and tranquility to patients during hospitalization, trying to supply problems reported by patients in various reported areas, working in stimulating communication skills. The introduction of medical students to the patient early encouraged the strengthening of the humanization process in the doctor-patient relationship.
\end{abstract}

Keywords: Education, Medical. Humanization of Assistance. Students, Medical.

\section{Introdução}

O exercício da medicina, desde seus primórdios, deve contar com uma boa relação médico-paciente (RMP), com fundamentos éticos centrados em princípios como atenção e compaixão, além da solidariedade humana, tendo em sua missão tarefas como confortar, escutar, olhar e tocar seu paciente ${ }^{1}$. Um dos componentes da RMP é a empatia, que apesar da dificuldade de sua definição conceitual, para o paciente ela é capaz de oferecer segurança e torná-lo mais colaborativo durante o processo de tratamento, aspecto fundamental para que se atinja a efetividade do processo terapêutico ${ }^{2}$. Um de seus conceitos pode ser definido como habilidade de poder vivenciar o que outra pessoa está passando como se estivesse no lugar dela, compreendendo as emoções,

Afiliação dos autores: ${ }^{\dagger}$ Acadêmica do Curso de Medicina, IC projeto de pesquisa e extensão, Universidade de Vassouras. RJ, Brasil. ORCID:https://orcid.org/0000-0002-8205-8112 ${ }^{\ddagger}$ Acadêmica do Curso de Medicina, IC projeto de pesquisa e extensão, Universidade de Vassouras. RJ, Brasil. ORCID: https://orcid.org/0000-0002-7305-3504

${ }^{\S}$ Acadêmico do Curso de Medicina, IC projeto de pesquisa e extensão, bolsista PIBIC CNPq,Universidade de Vassouras. RJ, Brasil. ORCID: https://orcid.org/0000-0003-4211-1887

" Acadêmica do Curso de Medicina, IC projeto de pesquisa e extensão, Universidade de Vassouras. RJ, Brasil. ORCID: https://orcid.org/0000-0002-4461-7470

${ }^{\circ}$ Coordenador do projeto de extensão, do Programa de Aproximação a Prática Médica 1 e e coordenador do curso de Medicina da Universidade de Vassouras. RJ, Brasil. ORCID https://orcid.org/0000-0002-9501-0353

${ }^{\square}$ Coordenadora do projeto de pesquisa e docente do Curso de Medicina do Programa de Aproximação a Prática Médica 1 da Universidade de Vassouras. RJ, Brasil. ORCID: https:// orcid.org/0000-0002-4295-0165.

* Email de correspondência: ivanapicone@globo.com

Recebido em: 31/03/20. Aceito em: 23/05/20. 
aflições e sentimentos dessa pessoa ${ }^{3}$.

O uso da empatia, assim como da humanização na medicina é capaz de fazer a reimplantação do afeto no cuidado, através da visão do profissional pelos dos olhos do paciente, proporcionando conforto durante seu processo de adoecimento e cuidado, dando a ele permissão para que possa decidir a respeito da conduta de seu tratamento ${ }^{4-5}$. Há também a correlação da empatia com a diminuição do estresse ao qual o profissional de saúde é submetido ${ }^{6}$.

É necessário a presença de uma equipe multidisciplinar na instituição do tratamento humanizado, objetivando uma análise física, social, psicológica e cultural do paciente ${ }^{5}$. Há uma preocupação pela equipe educacional de que ao longo da formação médica, esse treinamento faça com que os níveis de empatia declinem, como fator contribuinte as longas jornadas de estudo, os desafios da graduação e a privação de sono ${ }^{7}$. Foi demonstrado através de estudos que ocorre a diminuição da empatia ao longo da faculdade de medicina e residência médica, destaca-se que isso ocorre com maior frequência naqueles que escolhem especialidades com menor contato médico-paciente e que esse declínio ocorre ao entrar no ciclo clínico, onde há um maior contato com o paciente ${ }^{8}$.

Observa-se a necessidade da inserção de uma visão humanizada e da empatia nos cuidados em saúde na RMP, através de métodos de ensino onde, desde o primeiro ano da graduação possam garantir o desenvolvimento de aspectos humanísticos nos âmbitos de autonomia, participação e transformação, onde além do ensinamento teórico, possa ocorrer a aplicação prática ${ }^{9}$.

É visto que o desenvolvimento do aluno de medicina ao longo de sua formação prioriza essencialmente e principalmente o conhecimento biomédico, exigindo cada vez mais o desenvolvimento de sua capacidade cognitiva em detrimento ao seu lado afetivo e emocional ${ }^{10}$, dessa forma, indo em direção oposta ao que é proposto pela humanização.

A Política Nacional de Humanização (PNH) implantada no Sistema Único de Saúde (SUS), lançada em 2003, busca estimular a comunicação entre os funcionários do SUS e seus usuários de modo a buscar formas de enfrentar problemas e dificuldades, buscando resolvê-los de forma coletiva, com participação social ${ }^{11}$. Formação em saúde tem como pressuposto que haja a habilitação desse profissional em compreensão e comunicação com seu paciente, necessitando que ele seja capaz de compreender o ser humano no contexto que está inserido, seja ele social, econômico ou cultural e a partir disso, ser capaz de lidar com as singularidades de cada um ${ }^{12}$.

Aprimorar as relações interpessoais ao longo do curso de medicina é necessário e deve ser estimulado com a inserção da prática da humanização desde o princípio da vida acadêmica, possibilita que esse aprendizado se torne um hábito a ser seguido ao longo da vida profissiona ${ }^{13}$.

A medicina hoje é fragmentada por especialidades e acredita-se que isso possa ter contribuído para que o médico passasse a enxergar o paciente somente pelo aspecto da doença que ele possui, não se atentando para todo o aspecto psicológico e social que esse processo de adoecimento causa, levando o doente a pensar que o médico não é franco a respeito do que está acontecendo, trazendo a sensação de inferioridade causada pelo distanciamento entre médico e paciente, trazendo assim prejuízo direto na $\mathrm{RMP}^{13}$.

É preciso que se estabeleça um tratamento de forma humanizada, pautado na empatia e na individualização de cada paciente, oferecendo um tratamento personalizado, auxiliado por uma equipe multiprofissiona ${ }^{5-10}$. O objetivo do presente estudo foi avaliar os resultados de três anos evolutivos do Projeto "Calouro Humano", o qual aproximou os alunos do primeiro período de curso de medicina (APPCM) ao paciente com auxílio dos alunos matriculados a partir do segundo período do curso, incentivando a humanização da RMP.

\section{Métodos}

Realizou-se um estudo observacional e transversal, integrando o projeto de pesquisa inserido na Pró-Reitoria de Pesquisa e Pós-graduação da Universidade de Vassouras sob o parecer do Conselho de Ética em Pesquisa (CEP) n $n^{\circ} 1.963 .944$ com aprovação no ano de 2016, ao projeto de extensão vinculado a PróReitoria de Extensão Universitária da Universidade de Vassouras, com início em 2017 até a presente data.

Atuou com a inserção precoce do APPCM no ambiente hospitalar com auxílio de uma equipe de mentores representados pelos alunos matriculados a partir do $2^{\circ}$ período do curso de medicina - "ex-calouros humanos". Para avaliação e interpretação foi aplicado questionário anônimo, após assinatura do Termo de consentimento livre e esclarecido (TCLE), aos pacientes contendo 08 perguntas sobre sua internação hospitalar como: se tem sido informado sobre seu caso clínico; se há atendimento humanizado; o que espera encontrar no hospital durante seu tempo de permanência; se a internação traz algum transtorno a sua rotina pessoal com múltiplas opções de resposta - transtornos referentes a própria doença; com a comunicação com familiares; com o trabalho; financeiros; higiene pessoal; no que ele poderia ser ajudado.

Cada grupo de APPCM ficou responsável por um leito hospitalar pré-determinado supervisionados pelos alunos mentores e todos os pacientes que ali fossem internados, foram abordados pelo lado humano e não 
como portadores da doença.

\section{Resultados}

Foram entrevistados 2386 pacientes em internação hospitalar, através de questionário anônimo e executado por alunos do APPCM, durante os anos de 2017 a 2019. Onde encontrou-se que, ao serem perguntados sobre do grau de satisfação obtido a respeito do que lhes é informado sobre sua doença, $1.943(81,43 \%)$ afirmaram estar plenamente satisfeitos, $363(15,21 \%)$ estão parcialmente satisfeitos 79 (3,31\%) relataram insatisfação, e destes, $1(0,04 \%)$ não soube informar (Figura 1). Quando questionados a respeito da humanização do atendimento por toda a equipe de saúde, 2.311 (96,86\%) afirmaram estar recebendo atendimento humanizado, $68(2,85 \%)$ negaram e 7 $(0,29 \%)$ não informaram (Figura 2$)$. Do total, 1.651 $(69,2 \%)$ consideraram a participação dos alunos com enfoque humanizado, acompanhando os casos e colaborando assistencialmente com as necessidades pessoais dos pacientes como excelente, $636(26,66 \%)$ como boa, $78(3,27 \%)$ como regular e $14(0,59 \%)$ como ruim, havendo $7(0,3 \%)$ que não responderam (Figura 3 ). De modo a buscar maior conhecimento no que tange essas necessidades, foi perguntado o que ele espera encontrar no hospital durante sua internação, foram dadas 4 alternativas para que fosse assinalada a ordem de preferência do entrevistado. Em primeiro lugar $1.678(70,33 \%)$ afirmaram buscar a cura da doença, $255(10,69 \%)$ atendimento respeitoso, $208(8,72 \%)$ esperam que haja informações sobre a doença, 176 (7,38\%) buscam atenção dos profissionais de saúde e 69 $(2,89 \%)$ não informaram (Figura 4). Em segundo lugar $369(15,47 \%)$ buscam atendimento respeitoso, 250 $(10,48 \%)$ por atenção dos profissionais de saúde, 248 $(10,39 \%)$ por informações sobre a doença, $138(5,78 \%)$ a cura da doença e $1.381(57,88 \%)$ não informaram. Ainda pela ordem de preferência, em terceiro lugar,

\section{INFORMAÇÕES SOBRE O ESTADO DE SAÚDE}

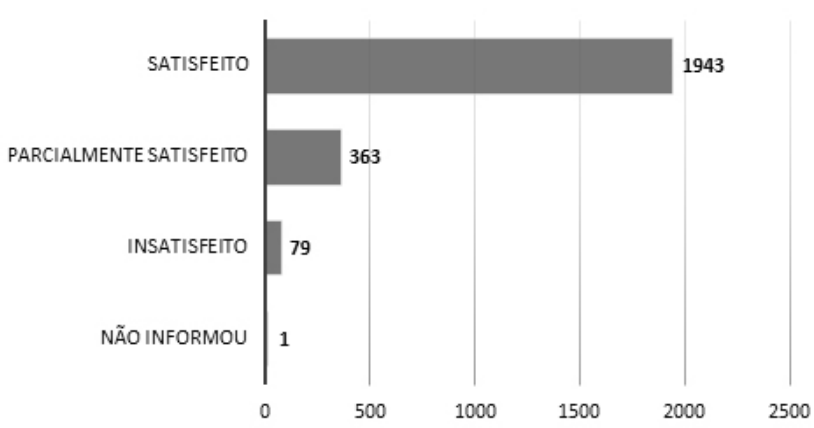

Figura 1. Percepção do paciente em relação as informações cedidas a respeito de sua doença
$350(14,67 \%)$ assinalaram atenção dos profissionais de saúde, $299(12,53 \%)$ atendimento respeitoso, 244 $(10,23 \%)$ informações sobre a doença, $83(3,48 \%)$ cura e $1.409(59,05 \%)$ não informaram. Em quarto lugar, $372(15,59 \%)$ solicitam informações sobre a doença, $290(12,15 \%)$ atenção dos profissionais, $216(9,05 \%)$ atendimento respeitoso, $88(3,69 \%)$ pela cura da doença e $1.420(59,51 \%)$ não informaram.

Um total de $1.025(42,96 \%)$ pacientes afirmaram estarem passando por transtornos em decorrência da hospitalização, 1.345 (56,37\%) negaram e $16(0,67 \%)$ não informaram (Figura 5). Entre o grupo que afirmou transtornos em decorrência da internação hospitalar, foram identificados: $457 \quad(17,18 \%)$ preocupação com a doença, $288(10,83 \%)$ referem problemas de comunicação com os familiares, $305(11,47 \%)$ com o trabalho, $216(8,12 \%)$ com problemas financeiros, $37(1,39 \%)$ com a higiene pessoal e $63(2,37 \%)$ não informaram (Figura 6).

Em relação a experiência relatada pelos APPCM, entre os 779 entrevistados, obteve-se que $625(80,23 \%)$ consideraram como ótima, $124(15,92 \%)$ como boa, 23 $(2,95 \%)$ como regular e $7(0,9 \%)$ como ruim (Figura 7). Quanto a aproximação proporcionada pelo projeto, $738(94,74 \%)$ relataram se sentirem mais próximos ao paciente, $39(5,01 \%)$ não identificaram alteração na relação entre o aluno e o paciente, $1(0,13 \%)$ relatou afastamento e 1 não informou (Figura 8). Sobre o auxílio que puderam proporcionar as necessidades do paciente, $584(74,97 \%)$ sentem ter atendido plenamente aos pedidos, $167(21,44 \%)$ parcialmente, $21(2,7 \%)$ afirmam não ter sido possível colaborar e $7(0,9 \%)$ não informou (Figura 9).

\section{Discussão}

Humanizar o atendimento médico decorre da necessidade de valorizar a dignidade do trabalhador e do usuário dos serviços de saúde, proporcionando um

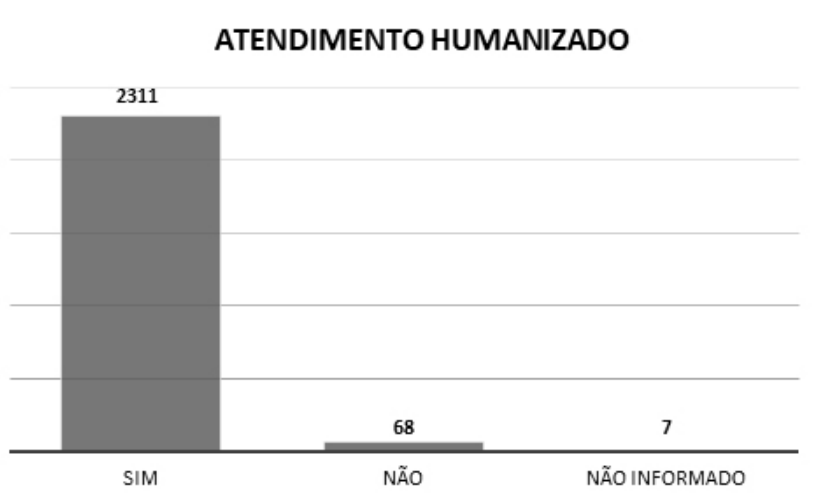

Figura 2. Percepção do a respeito da humanização do atendimento 


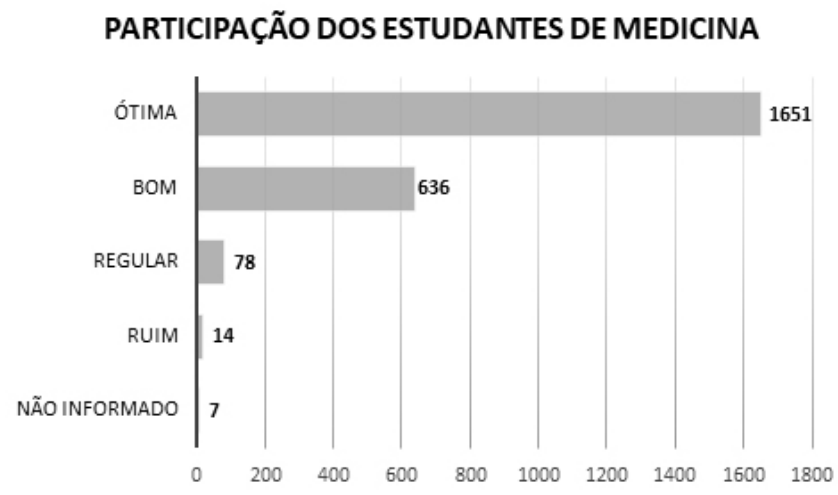

Figura 3. Opinião do paciente em relação a participação do estudante de medicina durante a internação

\section{O PRINCIPAL QUE O PACIENTE ESPERA ENCONTRAR HOSPITAL}

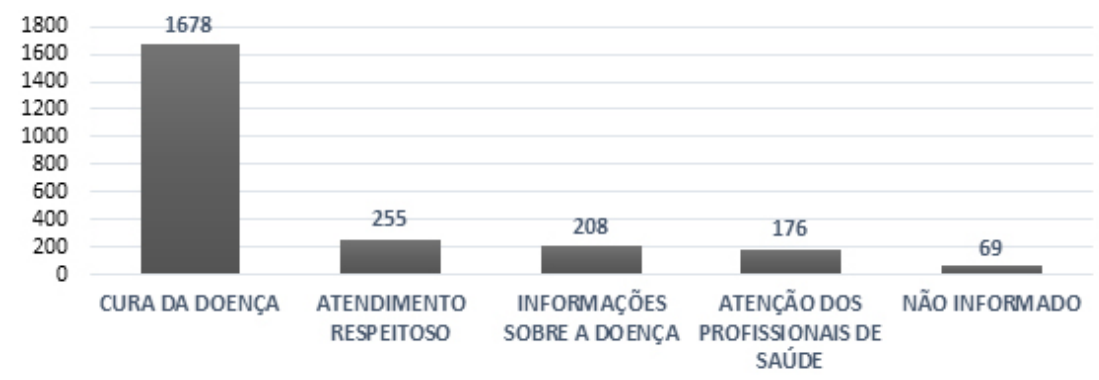

Figura 4. A primeira opção do que os pacientes esperam encontrar durante a internação

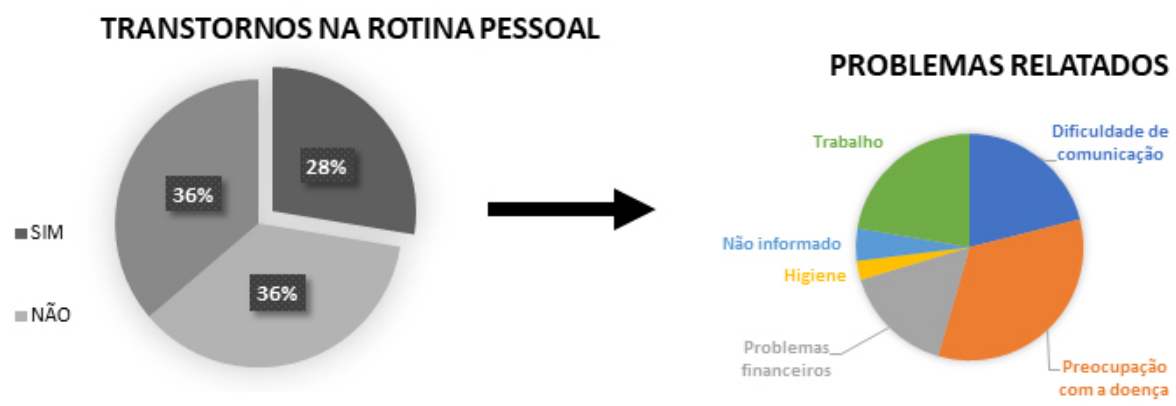

Figura 5 e 6. A percepção do paciente quanto aos transtornos causados em sua rotina devido a internação e quais são eles

EXPERIÊNCIA DOS APPCM

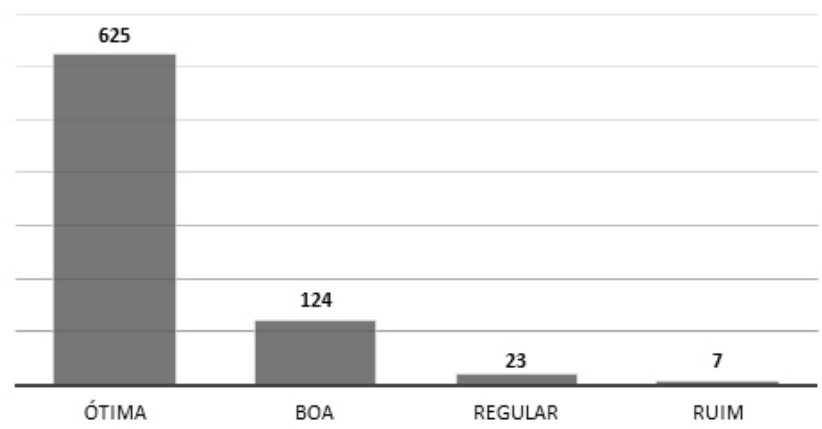

Figura 7. Opinião do aluno em participação no projeto
APROXIMAÇÃO COM O PACIENTE

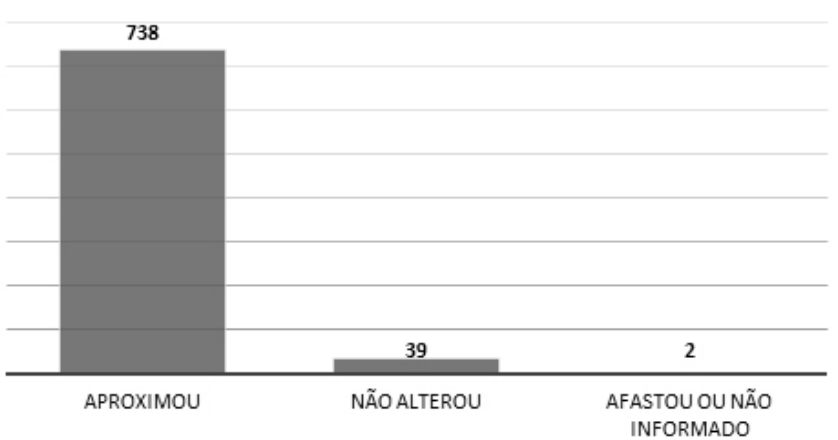

Figura 8. Percepção do aluno sobre o a aproximação com o paciente 


\section{APOIO AS NECESSIDADES DO PACIENTE}

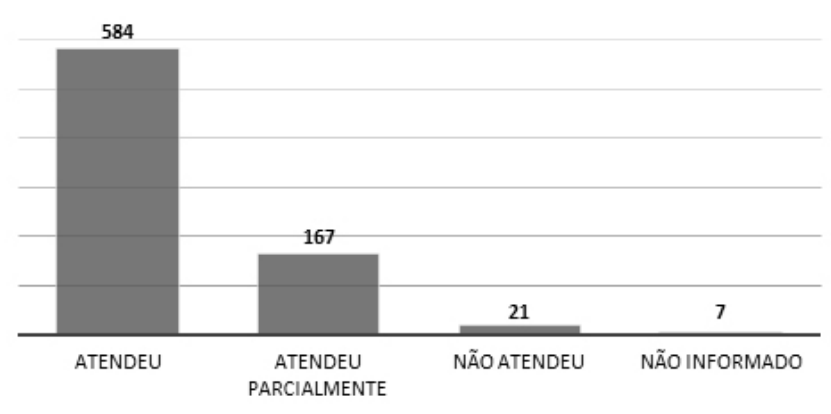

Figura 9. Percepção do aluno sobre o atendimento as necessidades do paciente.

atendimento baseado nos princípios do SUS como a integralidade, visando prestar assistência a todos, seja promoção, assistência ou reabilitação; equidade, para que possa haver a diminuição das desigualdades; sempre se pautando na universalidade, garantindo atenção à saúde para todos os cidadãos, conforme a assegura a Constituição Federal Brasileira e usando também a diretriz do SUS responsável pela participação social, que através de entidades representativas, participarão do processo de formulação e avaliação das políticas de saúde ${ }^{15 ; 16}$.

O profissional de saúde, devido ao contato direto com dor, sofrimento, insegurança e medo dos indivíduos portadores das doenças, pode vir a criar como mecanismo de defesa, um afastamento desse paciente, afetando a relação médico-paciente, portanto, humanizar o cuidado é uma estratégia de amenizar os efeitos desse distanciamento e poder dar início a reaproximação desse profissional com o seu paciente, sendo um processo demorado e complexo, não sendo generalizável pois é singular tanto para o médico quanto para o paciente e é muito importante para o processo de tratamento ${ }^{17}$. Proporcionar uma prática de conhecimento e construção da relação médico paciente pautada nos princípios da humanização desde o primeiro período de medicina possibilita que esse conhecimento seja algo sólido e natural, transformando esse estudante em um disseminador dessa prática ao longo de toda sua carreira médica. A importância de construir essa relação com o paciente provém de que caso esse paciente não se sinta acolhido e compreendido pelo profissional que o atende, onde é necessário ouvir as experiências, reflexões e crenças pessoais dessa pessoa, trabalhando a competência cultural e criando um vínculo, tornando assim o paciente mais colaborativo e positivo em relação a oferta de tratamento, pois caso ele se sinta desconfortável ou desrespeitado, tenderá a migrar para formas alternativas onde encontre esse acolhimento18;19;20. É parte do processo a oferta de integrar os avanços da tecnologia, o acolhimento, qualidade do atendimento e as condições de trabalho a que esse profissional está exposto ${ }^{21}$.

Ao perguntar a respeito dos transtornos que a internação causa em sua vida foi possível trabalhar no processo de entender o paciente como não somente a doença que ele possui, o entendendo como o ser biopsicossocial, onde problemas como financeiros, de comunicação, de necessidades básicas como higiene e a preocupação com a doença o afligem, tornando o tempo de hospitalização como algo potencialmente traumático se não houver a colaboração dos profissionais que estão prestando atendimento ${ }^{22}$.

\section{Considerações Finais}

A aproximação do aluno de primeiro período do curso de medicina ao paciente hospitalizado foi interpretada como positiva pelos alunos e pacientes, evidenciando que os estudantes sejam capazes de participar auxiliando no cuidado e fornecendo informações durante a internação, trazendo mais conforto e tranquilidade aos pacientes durante a internação, procurando suprir os problemas apresentados por eles, em várias áreas relatadas, trabalhando no estímulo da habilidade de comunicação. Portanto, o projeto "Calouro Humano" do curso de medicina da Universidade de Vassouras demonstrou que foi importante para ambos, alunos e pacientes, beneficiando a formação de profissionais potencialmente disseminadores desse conhecimento e proporcionando uma internação mais humanizada, trazendo maior efetividade ao tratamento.

Comitê de Ética: Aprovação pelo Conselho de Ética em Pesquisa (CEP) da Universidade de Vassouras - número 1.963.944

\section{Referências}

[1] Dórea AJPS. Relação médico x paciente. Disponível em: http://portal. cfm.org.br/index.php?option=com_content\&id=20464 Acesso em: 29 mar. 2020 .

[2] Costa F, Donizete D, Azevedo RCS. Empatia, relação médicopaciente e formação em medicina: um olhar qualitativo. Revista Brasileira de Educação Médica, 34(2), 261-269; 2010.

[3] Ruiz E. Empatia: Quero Olhar Pelo Seu Olhar. Disponível em: http:// redehumanizasus.net/88791-empatia-quero-olhar-pelo-seu-olhar/ Acesso em: 29 mar. 2020 .

[4] Sousa EA. Medicina Baseada em Empatia: vendo através do olhar do outro. Disponível em: http://redehumanizasus.net/medicina-baseada-emempatia-vendo-atraves-do-olhar-do-outro/ Acesso em: 29 mar. 2020.

[5] Humanização na medicina: a empatia pelo paciente. Disponível em: https://www.onelifealive.org/humanizacao-na-medicina-a-empatia-pelopaciente/ Acesso em: 29 mar. 2020.

[6] Neuwirth ZE. Physician empathy-should we care? The Lancet, 350 (9078), 606; 1997.

[7] Chen D, Lew R, Hershman W, Orlander J. A cross-sectional measurement of medical student empathy. Journal of General Internal 
Medicine, 22(10), 1434-1438; 2007.

[8] Neumann M, Edelhäuser F, Tauschel D, Fischer MR, Wirtz M, Woopen C, Scheffer C. Empathy Decline and Its Reasons: A Systematic Review of Studies With Medical Students and Residents. Academic Medicine, 86(8), 996-1009; 2011

[9] Corsino DLM, Sei MB. A Humanização nas grades curriculares de cursos da saúde de universidades públicas paranaenses. Revista Psicologia e Saúde, 11(1): 43-52; 2019.

[10] Rabello E, Bandeira LLB, Anjos ILPB, Santos CT, Macêdo TLS, Silva, CPO, Rebello DM, Dantas PRS, Soares RR, Neto ARB, Aragão IPB. Experiência inicial com a prática de humanização na relação médico-paciente em alunos do primeiro período do Curso de Medicina da Universidade de Vassouras. Revista Fluminense de Extensão Universitária 09 (1): 09-13; 2019.

[11] Ministério da Saúde. Política Nacional de Humanização. Disponível em: $\quad$ http://bvsms.saude.gov.br/bvs/publicacoes/politica nacional humanizacao_pnh_folheto.pdf Acesso em: 29 mar. 2020.

[12] Alves LS, Muhl C, Marce MM. Ensino médico e humanização: análise a partir dos currículos de cursos de medicina. Psicologia Argumento, 33(80); 2017.

[13] Anjos ILPB, Aragão IPB, Bandeira LLB, Marques SC, Santos, CT, Machado, RFS, Rabello, E. Relato da experiência acadêmica no projeto de Pesquisa e Extensão Universitária - "a prática da humanização da relação médico-paciente nos alunos de primeiro período de medicina da Universidade Severino Sombra. Projeto calouro humano". Revista Fluminense de Extensão Universitária 09 (2): 14- 16; 2019.

[14] Marques SC, Bandeira LLB, Anjos ILPB, Macedo TLS, Rebello DM, Rabello E, Aragão IPB. A prática da humanização da relação médicopaciente nos alunos de primeiro período de medicina da Universidade Severino Sombra: a visão do calouro que se tornou monitor- Um relato de experiência; 10 (2): 28-31; 2019.

[15] Casate JC, Corrêa AK, Humanização do atendimento em saúde: conhecimento veiculado na literatura brasileira de enfermagem. Revista Latino-Americana de Enfermagem 13(1):105-111; 2005.

[16] SUS: Princípios e diretrizes. Disponível em: https://siteantigo. portaleducacao.com.br/conteudo/artigos/medicina/sus-principios-ediretrizes/38572. Acesso em: 29 mar. 2020.

[17] Oliveira BRG, Collet N, Viera CS. A humanização na assistência à saúde. Revista Latino-Americana de Enfermagem; 14(2): 277-284; 2006

[18] Mota RA, Martins CGM, Véras RM. Papel dos profissionais de saúde na política de humanização hospitalar. Psicologia em Estudo, 11(2), 323$330 ; 2006$.

[19] Martins MCFN. Humanização da assistência e formação do profissional de saúde Disponível em: http://www.polbr.med.br/ano03/ artigo0503_1.php. Acesso em: 29 mar. 2020.

[20] Pusch R. Humanização e integralidade. Revista da Sociedade Brasileira de Psicologia Hospitalar; 13(2): 210-216; 2010.

[21] Garcia AV, Argenta CE, Sanches KR, São Thiago ML. Humanizar o humano: bases para a compreensão da proposição de humanização na assistência à saúde. Revista de Saúde Pública de Santa Catarina; 2 (1): 83$89 ; 2009$.

[22] Caprara A, Franco AL. A Relação paciente-médico: para uma humanização da prática médica [The patient-physician relationship: towards humanization of medical practice]. Cadernos de Saúde Pública; 15(3):647$654 ; 1999$. 